
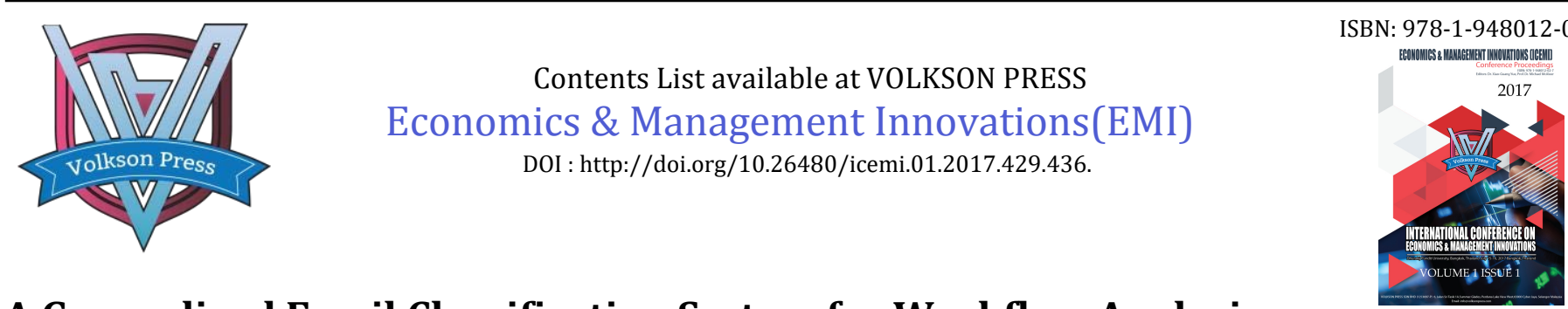

\title{
A Generalized Email Classification System for Workflow Analysis
}

\author{
Piyanuch Chaipornkaew ${ }^{1}$, Takorn Prexawanprasut ${ }^{1,}{ }^{*}$, Chia-Lin Chang ${ }^{2}$, Michael McAleer ${ }^{3}$ \\ ${ }^{1}$ College of Innovative Technology and Engineering, Dhurakij Pundit University, Bangkok, Thailand \\ 2Department of Applied Economics, Department of Finance, National Chung Hsing University, Taichung, Taiwan \\ ${ }^{3}$ Department of Quantitative Finance, National Tsing Hua University, Taiwan \\ Discipline of Business Analytics, University of Sydney Business School, Australia \\ Econometric Institute, Erasmus School of Economics, Erasmus University Rotterdam, The Netherlands \\ Department of Quantitative Economics, Complutense University of Madrid, Spain \\ Institute of Advanced Sciences. Yokohama National University, Japan \\ *Email: takorn.pre@dpu.ac.th \\ This is an open access article distributed under the Creative Commons Attribution License, which permits unrestricted use, distribution, and reproduction in any \\ medium, provided the original work is properly cited.
}

\section{ARTICLE DETAILS}

\section{Article History:}

Received 18 Disember 2017

Accepted 18 Disember 2017

Available online 19 Disember 2017

\section{Keywords:}

Email, business data, workflow management system, business transactions.

JEL: J24, 031, 032, 033.

\section{ABSTRACT}

One of the most powerful internet communication channels is email. As employees and their clients communicate primarily via email, much crucial business data is conveyed via email content. Where businesses are understandably concerned, they need a sophisticated workflow management system to manage their transactions. A workflow management system should also be able to classify any incoming emails into suitable categories. Previous research has implemented a system to categorize emails based on the words found in email messages. Two parameters affected the accuracy of the program, namely the number of words in a database compared with sample emails, and an acceptable percentage for classifying emails. As the volume of email has become larger and more sophisticated, this research classifies email messages into a larger number of categories and changes a parameter that affects the accuracy of the program. The first parameter, namely the number of words in a database compared with sample emails, remains unchanged, while the second parameter is changed from an acceptable percentage to the number of matching words. The empirical results suggest that the number of words in a database compared with sample emails is 11 , and the number of matching words to categorize emails is 7. When these settings are applied to categorize 12,465 emails, the accuracy of this experiment is approximately $65.3 \%$. The optimal number of words that yields high accuracy levels lies between 11 and 13, while the number of matching words lies between 6 and 8 .

\section{Introduction}

Information and communication technology has been developed significantly in recent years. The technology eliminates the wall of distance and connects people more closely than ever. The technology also supports many businesses to gain competitive advantages. Owing to this technology, large numbers of organizations are able to operate their business at lower costs and with a higher competitive advantage. As a result, many organizations attempt to acquire this on-time and accurate information. One of the most powerful tools in business is email, which is a fundamental and indispensable communication channel for every organization in the modern age.

In recent decades, the number of startup companies has increased dramatically. Two of the authors have participated in three start-up companies related to the import/export sector. These new start-ups established their own businesses by separating themselves from their former companies. After the initial study, it was found that startup companies needed to manage a large number of daily documents/emails because startup businesses contacted their customers and employees primarily via email. The employees also used these emails, which were stored in the mail server, as a database. For example, when employees wanted to find specific data, emails were the first place for seeking information.

In the first stage of starting their businesses, the number of emails was not large. However, when the scale of business expanded, the number of emails increased. The business owners needed applications to manage their company activities, a problem that could be solved primarily by software applications, such as the workflow management system. However, the cost of this software is rather high, and may not be appropriate for startup companies, so that alternative approaches to solve the problem were needed.

For the initial investigation, 12,465 of emails were selected from the three startup companies because they were written in English. As the employees in the selected companies wrote emails in two languages, namely English and Thai, only emails that were written in English were taken into consideration as the sentences in English are easier to separate into words than corresponding emails in Thai. By investigating some of these emails, some keywords specified the type of work, such as sales, transportation, billing, or shipping, which can be used as initial guidelines to conduct the classification models. 
The purpose of this paper is to define the categories of email and extract business data for a workflow management system.

The remainder of the paper is as follows. Section 2 provides a literature review, Section 3 describes the materials and methods, Section 4 presents the data analysis, Section 5 illustrates the results and discussion, and Section 6 provides some concluding comments.

\section{Literature Review}

There is much research that mentions the clustering and classification of email content, and many objectives to conduct research for email classification problem, such as: distinguishing between personal and machine-generated email [1]; classifying emails for contact centers [2]; classifying emails for automated service handling [3]; and classifying emails for social network analysis [4]. As regards classification techniques, there are also many methods applied to email classification, such as miningbased approaches [5], supervised learning algorithms [6], co-training technique [7,8], co-training with a Single Natural Feature Set [9], and regression-based approaches [10].

One of the interesting topics is by Alsmadia and Alhamib [11]. The authors illustrate that the best algorithm to perform email clustering and classification is NGram. Their sets of emails were in the form of a large text collection, which fits with the NGram algorithm, and the algorithm best fits the bi-language text. They conducted an experiment based on emails in both English and Arabic. The major challenge of their future work was that email servers or applications should include different types of pre-defined folders. The general pre-defined folders could be mailbox, sent, or trash, among others. Moreover, email servers or applications could allow users to add new folders for specific purposes, based on their NGram algorithm.

Further research on email classification is by Katakis, Tsoumakas, and Vlahavas [12]. They state that Machine Learning and Data Mining could be used as tools to automate email managing tasks, which could be far superior to other conventional solutions. They discuss the particularity of email content, and what special treatment it requires. In addition, there are some interesting email mining applications, like mail categorization, summarization, automatic answering, and spam filtering. In their experiments, they created an application to classify email based on several techniques, such as the Naïve Bayes Classifier and Support Vector Machines.

Ayodele, Khusainov, and Ndzi [13] present the design and implementation of a system to group, and summarize email messages. Their system considers the subject and content of email messages to classify emails based on user activities, and produces summaries of each incoming message with an unsupervised learning approach. They claim that their framework could solve the problems of email overload, congestion, difficulties in prioritizing, and difficulties in finding previously archived messages in the email server.

Another interesting topic is email grouping and summarization. Ayodele, Zhou, and Khusainov [14] present the design and implementation of an application to categorize and summarize email content. Their system extracts the subject and content of email messages for classification based on user activities to auto-generate a summary of each incoming message. They state that their framework could solve problems such as email overload, difficulties in prioritizing, and email congestion. Their framework also performs successful processing of new incoming messages.

Another interesting concept is automated email activity management, as in Kushmerick and Lau [15], who develop email applications that provide high-level support for structured activities in e-commerce. They define formal activities as finite-state automata, which correspond to the status of the process, and where transitions represent messages sent between participants. They propose several unsupervised machine learning algorithms, and evaluate a collection of e-commerce emails.

Schuff, Turetken, D'Arcy, and Croson [16] also discuss email classification. They implement effective e-mail management tools, which treat messages as useful information. This tool could economize on scarce cognitive resources at the expense of relatively cheap additional CPU power, disk capacity, and network bandwidth. In addition, they claim that their application provides automatic filtering, clustering, and a new user interface. Their system employs a large number of emails as an effective knowledge management tool, rather than as a source of information overload.

Email classification is discussed in Prexawantprasut and Chaipornkaew [17]. The research classifies email into four categories, namely sales, shipping, billing, and transportation. Two parameters are applied for the classification system, namely the number of words in a database compared with the sample emails, and an acceptable percentage to classify emails. The accuracy of classification is determined to be approximately $73.6 \%$.

Chaipornkaew, Prexawanprasut, and McAleer [18] discuss email extraction for workflow management system. In order to extract data, there are four criteria which are applied. Fifteen cases of alternative criteria to extract data are analyzed. The results show that when criteria numbers 2 and 4 are considered, email extraction accuracy is at the highest level. However, when the highest accuracy level occurs, the number of blanks fields is also high. According to user requirements, the number of blank fields should be at a low level. Therefore, the paper suggests that all four criteria should be considered to provide both an acceptable percentage of blank fields and also accuracy level.

\section{Materials and Methods}

The paper is planned in two phases, as shown in Figure 1. First, 1260 emails are selected randomly from the server to be used as training data for the system. These emails are 
then classified manually by employees into seven categories, namely (1) Sales, (2) Agent, (3) Shipping, (4) Customs, (5) Billing, (6) Packing and Moving, and (7) Insurance. The sentences in emails are separated into words, which are counted, as shown in Figure 2. These results are stored in the database, which is applied for email classification rules.

In order to test the defining rules, a further 12,465 emails are selected from the server. When these rules are accepted, the rest of the emails in the server are processed by the program. After the classification is processed, all emails are assigned to suitable categories, and then all the data are prepared for the second phase of the email classification system.

The second phase is to extract the classified emails, which are processed from the first phase. As in investigating the selected emails, there are key characteristics which can be represented as relationships. For example, the document number could be a key characteristic to define the relationships among the email messages. The program first reorders emails based on time in each category, then extracts data based on their characteristics. The final stage is to create a workflow management system from the extracted data.

\section{Data Analysis}

The first stage is to export all emails from the email server and format them in a text file, which is then imported to the program. The program first separates words in a text file. As the selected emails are in English, the algorithm to separate the words is the use of spaces. The words from the separation process are counted and stored in a database. The database stores all results which are all words, and their frequencies as shown in Table 1.

The research classifies 12,465 emails into seven categories, namely (1) Sales, (2) Agent, (3) Shipping, (4) Customs, (5) Billing, (6) Packing and Moving, and (7) Insurance. The mechanism is implemented based on the words found in emails compared with the words in the database for each category. Two parameters are considered in this experiment. The first parameter is the number of words in the database. For example, in order to gain greater accuracy in the classification, we need to determine whether the first 3 or 5 words in the database should be considered. The second parameter is the number of matching words that provides the highest accuracy to determine the category of email.

According to the data in Table 2, some email could not be classified because the number of matching words is less than the specified criteria. In this case, the second criterion is the first 5 words in a database. In order to obtain better results, these two criteria may need to be refined. As shown in Table 3, the first 10 words in a database are considered instead of the first 5 words.

The number of matching words is set at 5 in Table 2, and set at 4 in Table 3. As a result, only two groups of output in Tables 2 and 3 are the same. The first difference is the No. 2 group of emails. In Table 2, Email No. 2 could be either
Sales or Packing and Moving, but it is concluded to be Packing and Moving group in Table 3. The second difference is the No. 3 group of emails, which could not be grouped in Table 2, but could be defined as Agent in Table 3. The third difference is the No. 5 group of emails, which is defined as Insurance group in Table 2, while in Table 3 it is concluded to be Shipping.

The empirical data from both Tables 2 and 3 demonstrate that are two main factors that affect the grouping results. The first factor is the number of words in the database to be considered, while the second factor is the number of matching words. Therefore, another 12,465 emails are collected to test the program by changing the criteria for these two factors, with the empirical results shown in Figure 3.

\section{Results and Discussion}

The results shown in Figure 3 illustrate that the accuracy levels change when the number of words in the database and the number of matching words change. The purpose of the paper is to discover suitable parameter values, namely: (1) the number of words in the database to be considered; and (2) the number of matching words. The number of words in a database to be considered is adjusted from 5 to 20 , while the number of matching words are adjusted from 1 to 20 .

According to the results in Figure 3, the highest accuracy level of email classification occurs when the number of words in a database is 11 and the number of matching words is 7 . Therefore, these criteria are applied in the program. The program then classified the other 12,465 emails into seven groups, namely: (1) Sales, (2) Agent, (3) Shipping, (4) Customs, (5) Billing, (6) Packing and Moving, and (7) Insurance, as shown in Table 4.

According to Table 4, the program could not categorize all the emails because some emails do not meet the acceptable criteria. The program is able to define only 9,972 emails from a total of 12,465 emails, which represents $80 \%$ of the total. There are 2,493 emails which could not be categorized in the experiment. In order to improve the program efficiency, other factors could be concerned. One possible factor could be the importance level of each word (the weight of each word) in a database. For example, words that are found most frequently in emails should be placed at a higher level of importance than those that are found less frequently.

When the first phase is completed, all emails are already classified into groups (Sales, Agent, Shipping, Customs, Billing, Packing and Moving, and Insurance). The next phase is to analyze the characteristics of the emails. Key characteristics are defined by employees. The program collects these characteristics, which are applied for data extraction. The program reorders the events based on time in each category, as shown in Figure 4.

The last stage is to extract the specified data based on their characteristics. As the characteristics of data are in many forms, the extracted data can vary substantially. One 
example of data which are extracted based on Document Number (FW00018) is shown in Figure 5. According to the results, all the details concerned with Document Number (FW00018) are well summarized. The data that are extracted will be stored in a database, which will be implemented for a workflow management system.

\section{Conclusion}

According to the experiments, the accuracy level of email classification depends on two factors, namely the number of words to be considered in a database, and the number of matching words. After testing the program with different values for these two factors, the results show that the optimal value for the number of words in a database is 11 , while the number of matching words is 7 . The results also illustrate that high accuracy levels fall in the range of the number of words lying between 11 and 13, while the range of the number of matching words lies between 6 and 8 .

As mentioned earlier, the experiments select all emails in English, so some words need to be neglected. Examples of words which should not be considered are 'and', 'not', 'thanks', 'regards', and 'please'. As these words could be found in most emails, they should not be included in the program. As these words could not be used as criteria to classify email, a more sophisticated program should be developed to ignore these words before processing the email classifications.
In investigating email content, there are specific words that should not be used as criteria in email classification. Examples of these words are FREIGHTLINKS, STARSHIP, and HERMESINT'L. As these words are actual customer names, they should be defined as customer names in the database, and are excluded from the criteria for email classification in the first phase. However, these specific data are the key characteristics for the second phase of the research. The data with their characteristics are applied to extract data, which are used for the workflow management system.

The generalized email classification system for workflow analysis has been shown to work well in the experiments, with a high degree of accuracy.

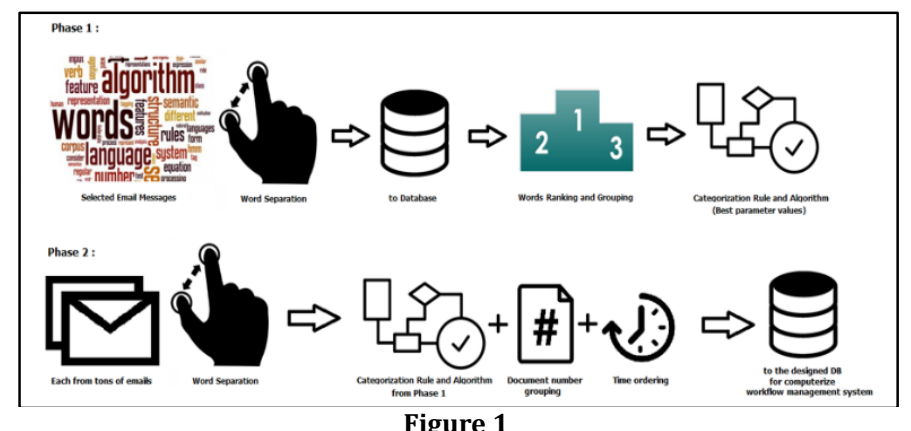

Two Phases of the Email Classification System

\begin{tabular}{|l|r|r|r|r|r|r|r|r|r|r|}
\hline & about & \multicolumn{1}{|c|}{ Above } & according & acknowledge adding & advise & after & \multicolumn{2}{l|}{ again } & ahead \\
\hline Sales & 4 & 1 & 2 & 3 & 1 & 1 & 0 & 0 & 2 \\
\hline Agent & 3 & 0 & 2 & 2 & 1 & 1 & 0 & 0 & 3 \\
\hline Shipping & 1 & 2 & 1 & 3 & 2 & 5 & 1 & 0 & 4 \\
\hline Customs & 0 & 0 & 2 & 1 & 4 & 2 & 0 & 1 & 1 \\
\hline Billing & 2 & 0 & 1 & 1 & 2 & 1 & 1 & 0 & 2 \\
\hline Packing and Moving & 2 & 3 & 2 & 1 & 1 & 2 & 0 & 1 & 2 \\
\hline Insurance & 1 & 0 & 1 & 0 & 2 & 3 & 1 & 0 & 1 \\
\hline
\end{tabular}

\begin{tabular}{|c|c|c|c|c|c|c|c|c|c|c|}
\hline & all & already & and & any & anytime & apply & around & arrange & Arrival & attached \\
\hline Sales & 2 & 1 & 2 & 1 & 2 & 0 & 3 & 1 & 5 & 8 \\
\hline Agent & 1 & 3 & 1 & 0 & 5 & 2 & 1 & 1 & 1 & 6 \\
\hline Shipping & 2 & 2 & 1 & 0 & 1 & 2 & 2 & 0 & 1 & 2 \\
\hline Customs & 1 & 2 & 0 & 1 & 3 & 3 & 2 & 0 & 3 & 7 \\
\hline Billing & 0 & 1 & 0 & 2 & 2 & 4 & 2 & 6 & 2 & 5 \\
\hline Packing and Moving & 3 & 0 & 1 & 3 & 1 & 4 & 4 & 2 & 4 & 4 \\
\hline Insurance & 2 & 1 & 2 & 1 & 2 & 1 & 1 & 7 & 1 & 3 \\
\hline
\end{tabular}

Figure 2

Example of Results from the Word Separation Process 


\begin{tabular}{|c|c|c|c|c|c|c|c|c|c|c|c|c|c|c|c|c|c|c|c|c|}
\hline \multirow{2}{*}{$\begin{array}{l}\text { Number } \\
\text { of Words }\end{array}$} & \multicolumn{20}{|c|}{ Number of Matching Words } \\
\hline & 1 & 2 & 3 & 4 & 5 & 6 & 7 & 8 & 9 & 10 & 11 & 12 & 13 & 14 & 15 & 16 & 17 & 18 & 19 & 20 \\
\hline 5 & 13.7 & 27.9 & 30.6 & 30.1 & 29.9 & & & & & & & & & & & & & & & \\
\hline 6 & 14.4 & 20 & 24 & 32.1 & 32.5 & 33.1 & & & & & & & & & & & & & & \\
\hline 7 & 18.2 & 19.5 & 20.1 & 23.6 & 36.8 & 32.3 & 23.3 & & & & & & & & & & & & & \\
\hline 8 & 16.2 & 17.5 & 18.2 & 32.5 & 40.1 & 25.2 & 20.1 & 18.3 & & & & & & & & & & & & \\
\hline 9 & 15.2 & 23.6 & 38.8 & 42.6 & 42.1 & 39.6 & 35.3 & 35.1 & 23.5 & & & & & & & & & & & \\
\hline 10 & 19.2 & 18.2 & 35.5 & 48.5 & 47.8 & 50.1 & 38.5 & 26.5 & 28.2 & 20.5 & & & & & & & & & & \\
\hline 11 & 14.6 & 23.5 & 28 & 36.3 & 45.1 & 56.8 & 65.3 & 52.2 & 35.1 & 18.2 & 10.8 & & & & & & & & & \\
\hline 12 & 15.2 & 18.5 & 20.4 & 25.7 & 40.6 & 58 & 58.1 & 60.1 & 39 & 20.3 & 18.8 & 12.8 & & & & & & & & \\
\hline 13 & 18.7 & 19.6 & 21.5 & 26 & 47.5 & 63.3 & 54.9 & 62.1 & 41 & 25.1 & 19.5 & 15.1 & 15.6 & & & & & & & \\
\hline 14 & 14.3 & 20.1 & 12.1 & 15.6 & 20.7 & 32.2 & 34 & 45.6 & 28.9 & 29.4 & 18.8 & 12.3 & 10.2 & 8.75 & & & & & & \\
\hline 15 & 14.5 & 15.7 & 13.3 & 12.1 & 15.5 & 18.3 & 20.3 & 14.5 & 13.3 & 12.5 & 10.8 & 8.45 & 8.5 & 7.85 & 12.3 & & & & & \\
\hline 16 & 12 & 19 & 15.7 & 13.2 & 12.6 & 11.9 & 10.3 & 18.5 & 15.2 & 12.3 & 14.6 & 13.8 & 17.4 & 12.1 & 11.5 & 10.6 & & & & \\
\hline 17 & 6.02 & 14.6 & 12.3 & 11.9 & 10.6 & 8.72 & 14.3 & 13.2 & 12.6 & 11.6 & 13.2 & 17.6 & 15.5 & 13.2 & 11.2 & 9.25 & 10.8 & & & \\
\hline 18 & 10.6 & 12 & 10.2 & 8.92 & 9.95 & 8.75 & 12.2 & 15.3 & 10.8 & 18.5 & 12.2 & 12 & 8.16 & 7.13 & 10.6 & 7.75 & 10.2 & 9.5 & & \\
\hline 19 & 8.12 & 9.87 & 10.2 & 13.9 & 10.3 & 13.8 & 15.5 & 18 & 15.9 & 13.5 & 13 & 13.3 & 14.1 & 8.54 & 12.3 & 8.63 & 8.72 & 9.58 & 9.98 & \\
\hline 20 & 9.5 & 7.14 & 12.5 & 8.12 & 10.2 & 11.5 & 15.5 & 13.9 & 12.5 & 11.6 & 10.1 & 13.5 & 12.2 & 10.3 & 5.56 & 6.23 & 8.59 & 6.54 & 7.63 & 9.72 \\
\hline
\end{tabular}

Figure 3

\section{Accuracy (\%) of Email Classification}

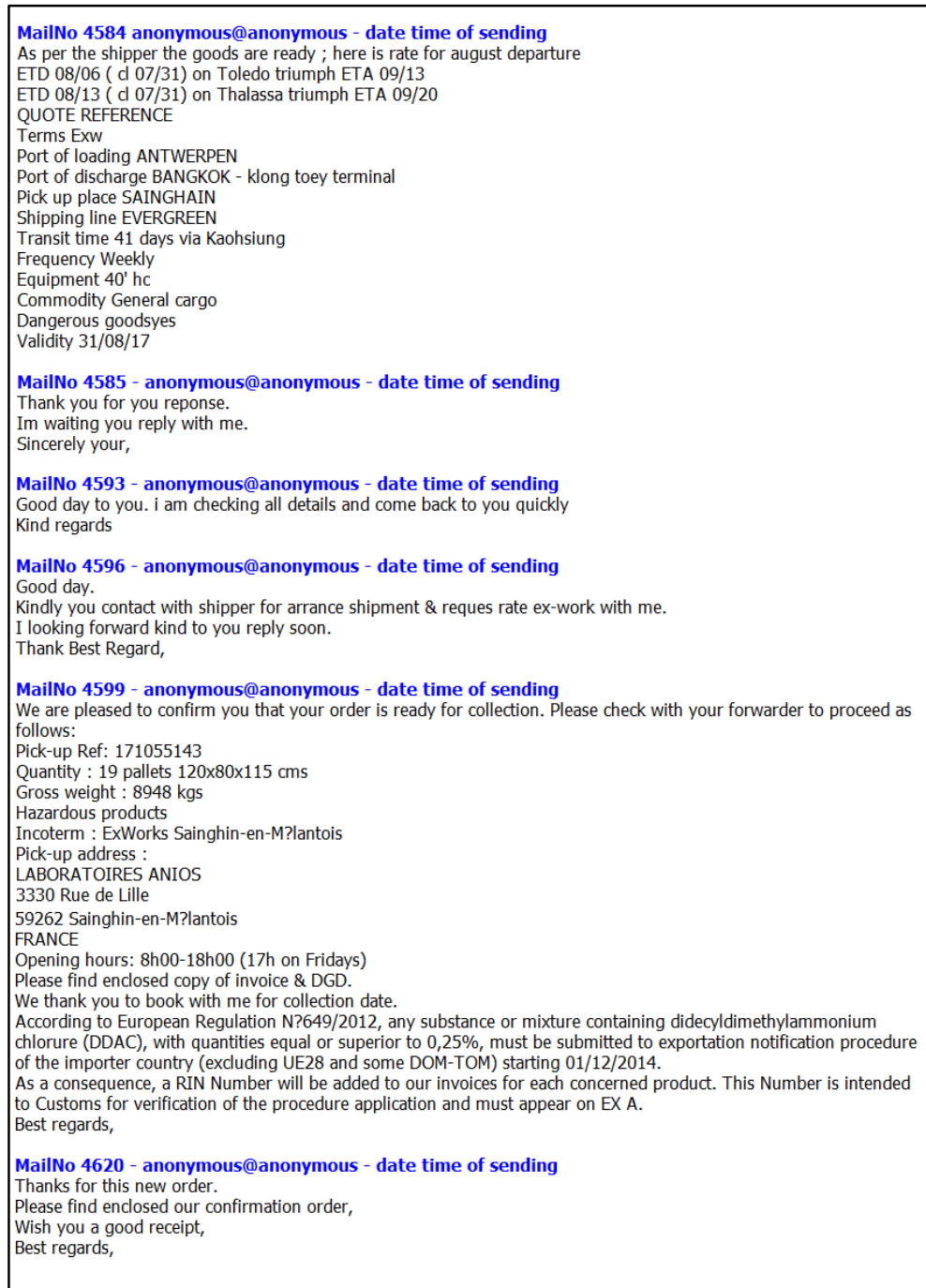

Figure 4

Program Results after Grouping, Event Ordering, and Inclusion of Email Characteristics 
Related issues from mail number $4281,4292,4297,4320 . .$.

Document Number 1 : FW00018-00008

Document Number 2 : FW00018-00017

Document Number 3 : FW00018-00019

add related documents

Icon Global Logistics / H020-1600078

add related participants

\begin{tabular}{|c|c|}
\hline Shipper: & CHIEF \\
\hline Consignee: & POP MEDIC \\
\hline PO No: & MKC 01-2017 \\
\hline Volume : & 46 cartons $/ 549.8 \mathrm{~kg} / 4$. \\
\hline Vessel/voy : & NORDOCELOT V-201Q \\
\hline Consignee: & 3days \\
\hline sing date & $17 / 07 / 14$ \\
\hline & $17 / 07 / 18$ \\
\hline & $17 / 07 / 25$ \\
\hline
\end{tabular}

add related events

Remark Subject to local charge both of side

add related remarks

Figure 5

Example of Extracted Data from Phase 2, Based on Document Number

Table 1

Top 15 Words in Emails in 7 Categories

\begin{tabular}{|c|c|c|c|c|c|c|c|}
\hline \multicolumn{2}{|c|}{ Sales } & \multicolumn{2}{|c|}{ Agent } & \multicolumn{2}{|c|}{ Shipping } & \multicolumn{2}{|c|}{ Customs } \\
\hline Word & Frequency & Word & Frequency & Word & Frequency & Word & Frequency \\
\hline agent & 112 & \#NAME of CUS & 188 & shipment & 167 & $\operatorname{tax}$ & 109 \\
\hline volume & 91 & arrange & 165 & scheduled & 112 & standard & 87 \\
\hline \#NAME of CUS & 88 & ETA & 150 & ETA & 102 & customs & 74 \\
\hline product & 72 & delivery & 112 & \#Date format & 89 & clear & 52 \\
\hline shipment & 60 & \#NAME of CITY & 94 & ship & 82 & \#Date format & 43 \\
\hline \#NAME of CITY & 58 & import & 86 & $\mathrm{D} / \mathrm{O}$ & 80 & scheduled & 42 \\
\hline process & 55 & items & 81 & shipper & 65 & \#NAME of port & 38 \\
\hline confirm & 52 & \#NAME of PORT & 75 & \#NAME of CUS & 55 & shipment & 33 \\
\hline week & 48 & warehouse & 53 & \#NAME of CITY & 42 & departed & 28 \\
\hline \#Date format & 31 & service & 50 & confirm & 40 & \#NAME of PORT & 25 \\
\hline $\mathrm{D} / \mathrm{O}$ & 28 & update & 48 & HBL & 35 & fare & 23 \\
\hline packing list & 25 & port & 39 & BL & 32 & transaction & 22 \\
\hline \#NAME of PORT & 22 & shipping & 31 & port & 21 & notification & 18 \\
\hline attach & 18 & scheduled & 21 & \#NAME of PORT & 19 & standard & 16 \\
\hline request & 16 & \#Date format & 12 & request & 17 & arrived & 15 \\
\hline
\end{tabular}




\begin{tabular}{|c|c|c|c|c|c|}
\hline \multicolumn{2}{|c|}{ Billing } & \multicolumn{2}{|c|}{ Packing and Moving } & \multicolumn{2}{|c|}{ Insurance } \\
\hline Word & Frequency & Word & Frequency & Word & Frequency \\
\hline consignee & 125 & loading & 108 & policy & 78 \\
\hline shipper & 111 & destination & 75 & dividend & 62 \\
\hline document & 94 & package & 71 & product & 55 \\
\hline revise & 89 & carrier & 60 & fair & 53 \\
\hline \#NAME of CUS & 84 & loader & 65 & \#NAME of CUS & 42 \\
\hline scheduled & 74 & \#Date format & 55 & accident & 41 \\
\hline departed & 62 & co-loader & 48 & rate & 28 \\
\hline service & 50 & departed & 40 & title & 24 \\
\hline \#Date format & 48 & ETD & 34 & revenue & 22 \\
\hline arrived & 42 & arrived & 33 & package & 22 \\
\hline \#NAME of PORT & 38 & scheduled & 33 & \#Date format & 21 \\
\hline shipment & 31 & \#NAME of PORT & 32 & arrived & 13 \\
\hline notice & 25 & shipment & 28 & departed & 13 \\
\hline booking & 22 & worker & 24 & loss & 11 \\
\hline approval & 18 & condition & 15 & value & 10 \\
\hline
\end{tabular}

Table 2

Grouping Results Based on Top 5 Words and 5 Acceptable Number of Matching Words

\begin{tabular}{|c|c|c|c|c|c|c|c|c|}
\hline \multirow[b]{2}{*}{ No. of Emails } & \multicolumn{7}{|c|}{ Number of Matching Words } & \multirow[b]{2}{*}{ Grouping result } \\
\hline & Sales & Agent & Shipping & Customs & Billing & $\begin{array}{l}\text { Packing } \\
\text { and } \\
\text { Moving }\end{array}$ & Insurance & \\
\hline 1 & 5 & 3 & 0 & 2 & 1 & 1 & 0 & Sales \\
\hline 2 & 5 & 0 & 4 & 1 & 1 & 5 & 1 & Sales or Packing and Moving \\
\hline 3 & 0 & 0 & 1 & 2 & 0 & 1 & 2 & Uncategorized \\
\hline 4 & 1 & 0 & 2 & 3 & 5 & 0 & 0 & Billing \\
\hline 5 & 1 & 0 & 4 & 0 & 3 & 2 & 5 & Insurance \\
\hline
\end{tabular}

Note: In the case of email no. 2, it falls into either Sales or Packing and Moving category. The research could not conclude whether it should be in the Sales or Packing and Moving group. This issue should be clarified in future research.

Table 3

Grouping Results Based on Top 10 Words and 4 Acceptable Number of Matching Words

\begin{tabular}{ccccccccc}
\hline \multirow{2}{*}{$\begin{array}{c}\text { No. of } \\
\text { Emails }\end{array}$} & Sales & Agent & Shipping & Customs & Billing & $\begin{array}{c}\text { Packing } \\
\text { and } \\
\text { Moving }\end{array}$ & Insurance & Grouping result \\
\hline 1 & 6 & 2 & 4 & 2 & 1 & 1 & 0 & Sales \\
2 & 4 & 0 & 2 & 1 & 1 & 5 & 1 & Packing and Moving \\
3 & 0 & 4 & 1 & 2 & 0 & 1 & 3 & Agent \\
4 & 1 & 0 & 4 & 4 & 7 & 0 & 0 & Billing \\
5 & 1 & 4 & 8 & 0 & 3 & 2 & 1 & Shipping \\
\hline
\end{tabular}


Table 4

Number of Emails in Each Category

\begin{tabular}{cccccccc}
\hline Sales & Agent & Shipping & Customs & Billing & $\begin{array}{c}\text { Packing } \\
\text { and Moving }\end{array}$ & $\begin{array}{c}\text { Insurance } \\
\text { Total }\end{array}$ \\
\hline 1,994 & 1,623 & 1,246 & 1,121 & 1,371 & 1,745 & 872 & 2,493 \\
\hline
\end{tabular}

\section{References}

[1] Mihajlo, G., Halawi, G., Karnin, Z., and Maarek, Y. (2014), How Many Folders Do You Really Need? Classifying Email into a Handful of Categories, Proceedings of the 23rd ACM International Conference on Conference on Information and Knowledge Management, November 2014, pp. 869-878.

[2] Nenkova, A., and Bagga, A. (2003), Email Classification for Contact Centers, Proceedings of the 2003 ACM Symposium on Applied Computing, March 2003, pp. 789-792.

[3] Taliby, R., Dean, R., Milner, B., and Smith, D. (2006), Email Classification for Automated Service Handling, Proceedings of the 2006 ACM Symposium on Applied Computing, April 2006, pp. 1073-1077.

[4] Yelupula, K., and Ramaswamy, S. (2008), Social Network Analysis for Email Classification, Proceedings of the 46th Annual Southeast Regional Conference on XX, March 2008, pp. 469-474.

[5] Aery, M., and Chakravarthy, S. (2004), EMailShift: Mining-based Approaches to Email Classification, Proceedings of the 27th Annual International ACM SIGIR Conference on Research and Development in Information Retrieval, July 2004, pp. 580-581.

[6] Tam, T., Ferreira, A., and Lourenco, A. (2012), Automatic Foldering of Email Messages: A Combination Approach, Proceedings of the 34th European Conference on Advances in Information Retrieval, March 2012, pp. 232-243.

[7] Kiritchenko, S., and Matwin, S. (2001), Email Classification with Cotraining, Proceedings of the 2001 Conference of the Centre for Advanced Studies on Collaborative Research, October 2001, pp. 1-10.

[8] Kiritchenko, S., and Matwin, S. (2011), Email Classification with Cotraining, Proceedings of the 2011 Conference of the Center for Advanced Studies on Collaborative Research, November 2011, pp. 301-312.

[9] Chan, J., Koprinska, I., and Poon, J. (2004), Co-training with a Single Natural Feature Set Applied to Email Classification, Proceedings of the 2004 IEEE/WIC/ACM International Conference on Web Intelligence,
September 2004, pp. 586-589.

[10] Yoo, S., Yang, Y., and Carbonell, J. (2011), Modeling Personalized Email Prioritization: Classification-based and Regression-based Approaches, Proceedings of the 20th ACM International Conference on Information and Knowledge Management, October 2011, pp. 729-738.

[11] Alsmadia, I. and Alhamib, I. (2015), Clustering and Classification of Email Contents, Journal of King Saud University - Computer and Information Sciences, 27(1), 46-57.

[12] Katakis, I., Tsoumakas, G., and Vlahavas I. (2006), E-mail Mining: Emerging Techniques for E-Mail Management, Web Data Management Practices: Emerging Techniques and Technologies, Idea Group Publishing, 2006, 220-243.

[13] Ayodele, T., Khusainov, R., and Ndzi, D. (2007), Email Classification and Summarization: A Machine Learning Approach, IET Conference on Wireless, Mobile and Sensor Networks (CCWMSN07). 2007, pp. 805-808.

[14] Ayodele, T., Zhou, S., and Khusainov, R. (2009), Email Grouping and Summarization: An Unsupervised Learning Technique, WRI World Congress on Computer Science and Information Engineering, 2009, pp. 575-579.

[15] Kushmerick, N. and Lau, T. (2005). Automated Email Activity Management: An Unsupervised Learning Approach, Proceedings of the 2005 International Conference on Intelligent User Interfaces, 2005, pp. $67-$ 74

[16] Schuff, D., Turetken, O., D'Arcy, J., and Croson, D. (2007), Managing EMail Overload: Solutions and Future Challenges, Computer, 40(2), 31-36.

[17] Prexawanprasut, T. and Chaipornkaew, P. (2017), Email Classification Model for Workflow Management Systems, Walailak Journal of Science and Technology, 14(10), 783-790.

[18] Chaipornkaew, P., Prexawanprasut, T., and McAleer, M. (2017), You've Got Email: A Workflow Management Extraction System, Journal of

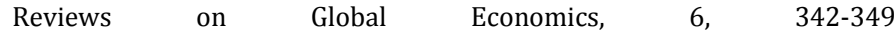

